\title{
Smart Pharmacy Kanban System in Post Covid Era
}

\author{
Shymalagowri Selvaganapathy ${ }^{1}$, Hema Priya $\mathrm{N}^{2}$ \\ \{shymalagowri@gmail.com ${ }^{1}$,nhp.it@psgtech.ac.in $\left.{ }^{2}\right\}$
}

Assistant Professor, Department of Information Technology, PSG college of Technology, Coimbatore ${ }^{1,2}$, india.

\begin{abstract}
Maintaining social distancing is crucial in all aspects of life in this pandemic situation. Stock updation is an important aspect in pharmacy to provide effective supply chain with the use of sensors. The availability of appropriate stock ensures that the product can be delivered to customer on time. In the advent of Enterprise Management System (ERM), most hospitals are integrating these software systems. However, manual effort is required for the updation of stocks in the ERM software which results in overhead costs such as payroll. In the existing model, if some of the old stocks are stocked for long time, it may lead to the waste of stocks. In order to avoid all of above problems, a Kanban planning based model has been built to implement the stock updation in pharmacy where all the hospitals can be benefited by reducing the manual work. This system also reduces the inadvertent errors caused by the workers and the number of employees and provide more effective supply chain to the hospital. It can enable designed to maintain the social distancing of pharmacists also enabling better storage their oxygen cylinders and vaccines in a particular place.
\end{abstract}

Keywords: RFID-Radio Frequency identification, ERP-Enterprise Re- source planning, SIM-Subscriber Identification Module, GPRS-General Packet Radio Service, TCP-Transmission control protocol, PCBPrinted Circuit Board, FTP-File Transfer Protocol.

\section{Introduction}

The Japanese word Kanban, which translates to 'shield' is synonymous with de- mand planning. Kanban has its roots in the early days of the Toyota production system [1]. In the late 1940s, Taiichi Onho developed Kanbans to control inter- process production and implement just-in-time (JIT) manufacturing at Toyota's manufacturing facilities in Japan. Using Kanban leads to minimized work be- tween processes and reduced inventory costs. In Kanban planning, operators use visual cues to determine how much to run and when to stop or recondition. Finally, a well-planned kanban has visual indicators that allow managers and supervisors to see the status of the line schedule at a glance. The example of a traditional Kanban card is shown in Table 1. Kanban rules also tell operators what to do when they have problems and who to contact when those problems arise. Kanban rules also tell the operators what to do, when they have problems and who to go when these problems arise. It has important details which include item's part number, item, name, supplier name, Vendor's details, storage details and location details. There are different varieties of kanban card used in various industries. Toyota's Kanban approach has been further developed and imple- mented in various settings. Existing models for stock maintenance needs lot of manual inspection. This article attempts to incorporate the benefits of kanban into the stock maintenance process and provide benefits in this post covid era.

The rest of the article is encompassed as Usage of kanban based systems in the recent inventory systems is detailed in Section 2. Section 3 provides work- ing of the proposed model along with its effectiveness while Section 4 provides conclusion and future improvements to work with real-time medical data. 
Table 1. Kanban Card

\begin{tabular}{lr} 
Part number & $80800-14898$ \\
\hline Part name & 10V power supply \\
Supplier & Smith electronics \\
Vendor number & 133344 \\
Container type & $12 \times 14$ \\
Container quantity & 20 \\
Delivery interval & Daily \\
Storage location & Portable radio line \\
Production operation & 40 \\
Bin location & $\mathrm{C}-3$ \\
Delivery location & Dock 4 \\
\hline
\end{tabular}

\section{Literature Survey}

A few practical E-Kanban systems devised to replace the traditionally manual card system previously used is discussed below.

In [1], the goal was to reduce the number of delays in supply of rubbers. The number of rubber supplying delays is reduced from 86 events to 12 events. [2] demonstrates how Kanban can be used effectively to exercise inventory controls. The implementation of the Kanban system has helped the pressure vessel department to know the status of the sub inventory. Cloud based Kanban framework [3]

enables better and faster systems than traditional systems. They provide flexibility to scale as demand increases, lowering total cost of ownership.

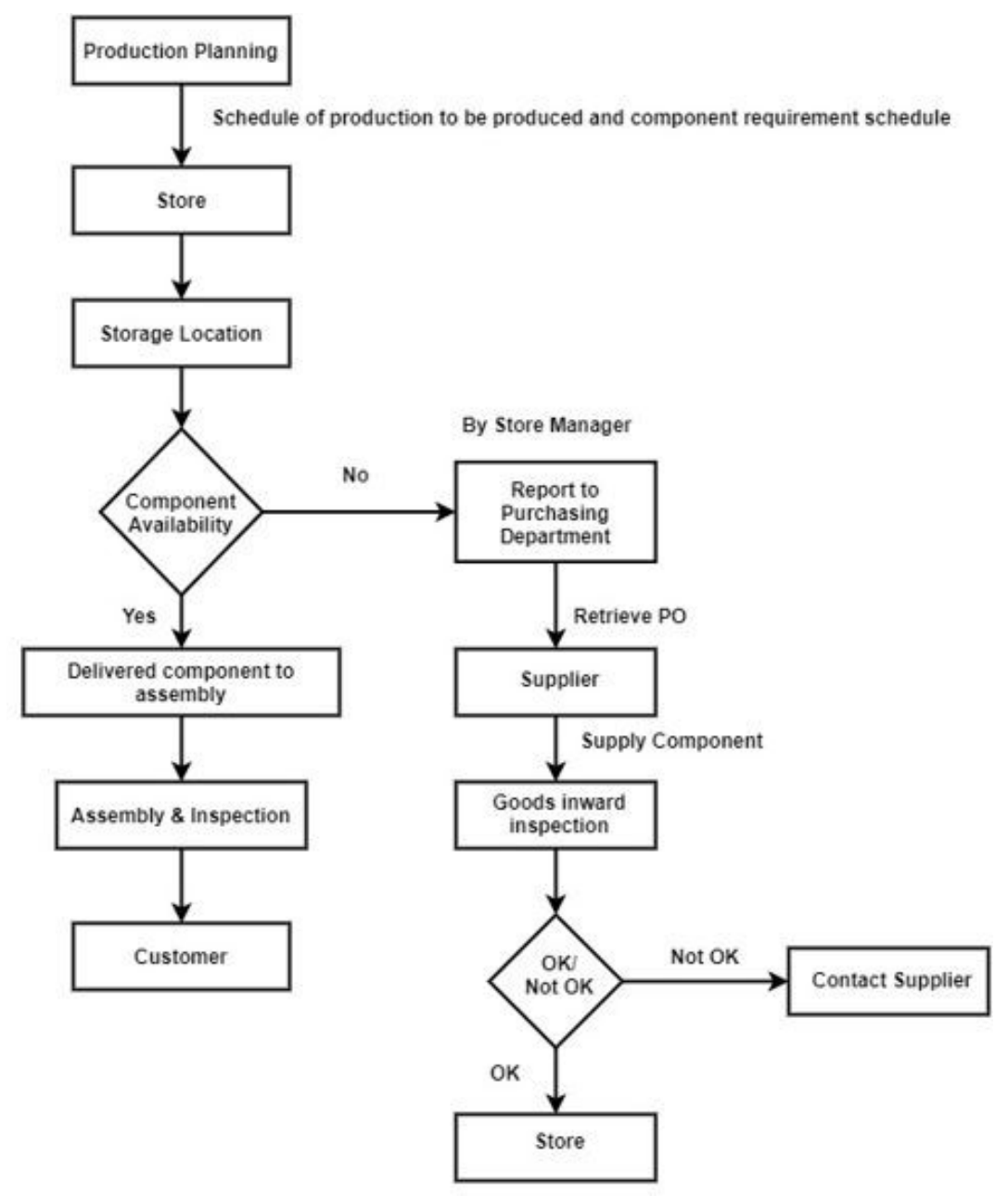

Fig. 1. Existing Pharmacy Stock Updation Model 
Attendance System using Kanban logic in [4] provides flexibility to detect the presence of students or users. The system provides an efficient, simple and easy-to-use user interface with high precision security. Raspberry Pi and Arduino were effectively used to design and implement the proposed attendance system based on radio frequency sensing and GSM technologies.

E-Kanban to Traditional kanban systems is compared in [5] where Electronic kanban cards developed makes the document management system easier, user- friendly, and also bears low maintenance cost.

Lean Production and Industry 4.0 were presented in [6] to show the possibility of combining these two approaches. A smart RFID-based product allows tagged Kanban cards to be recognized in real time. [7] demonstrates the elimination of manual work with the use of RFID and RASPBERRY PI to monitor students or staff members.

The above literature review enables to understand about the current trends and available technologies, and based on that, a concept for the solution can be created for pharmacy systems.

The existing pharmacy systems required lot of manual works for stock inspection and stock updation in the storage place of medicines. And also, it required high skilled labor for arranging right items in right locations. The existing model is shown in figure 1.

\section{Proposed Methodology}

The considered proposed model as given in figure 2 eliminates the manual stock updation by automatically placing an order, when the medicine falls to reorder level as shown in figure Stock module is very useful in reduction of man power which results in reduction of liability. It also eliminates the inadvertent errors caused by the worker by placing right items in right locations. It displays the right location for the medicine to be stored and also displays the path to reach that location. Finally, it helps the worker to find the first-in medicine during unloading the medicines for assembly. The individual modules developed such as Stock module, Locate module, FIFO module along with Alert module as shown in figure 3, figure 4, figure 5 and figure 6 respectively.

\subsection{Locate Module}

In almost all the mass production industries have huge number of racks for placing the medicines in their pharmaceutical storage place. Only skilled worker will be allowed for loading the particular medicines in their pharmacy stores. But, by using our locate module, even an unskilled labour can locate the right place in which the medicine should be placed as shown in figure 4 , when the respected medicines entered into the stores, RFID reader reads the tags and then in LCD display, the right location will be displayed.

\subsection{Alert Module}

Even though locate module displays the rack location, there may be chance that worker will misplacing the medicines in the rack. For this, Alert module is used is eliminating this error. The working of Alert module in shown in Fig 6, where the LED bulbs are placed in each cell. When the medicines entered into the stores, 


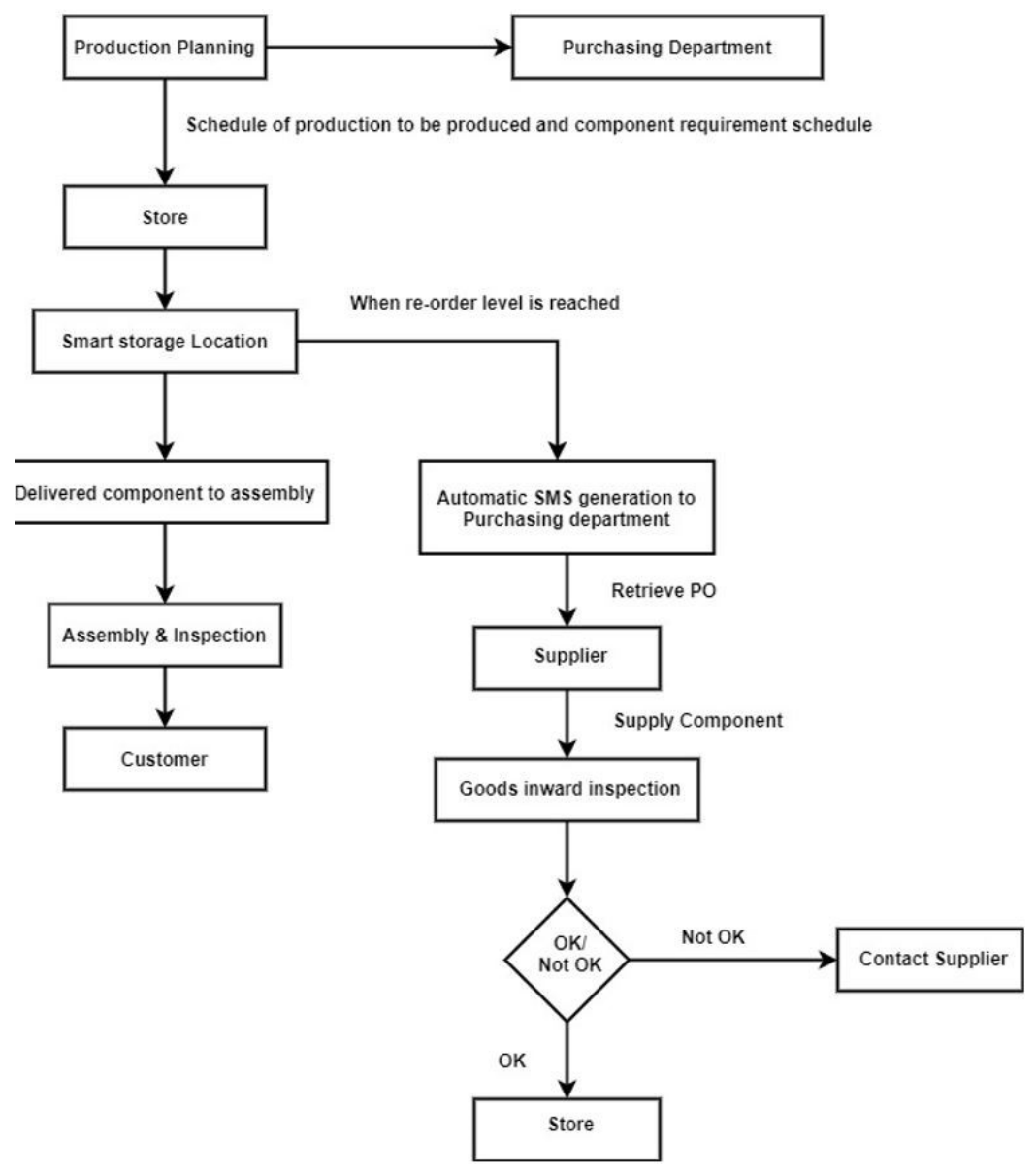

Fig. 2. Proposed Model 


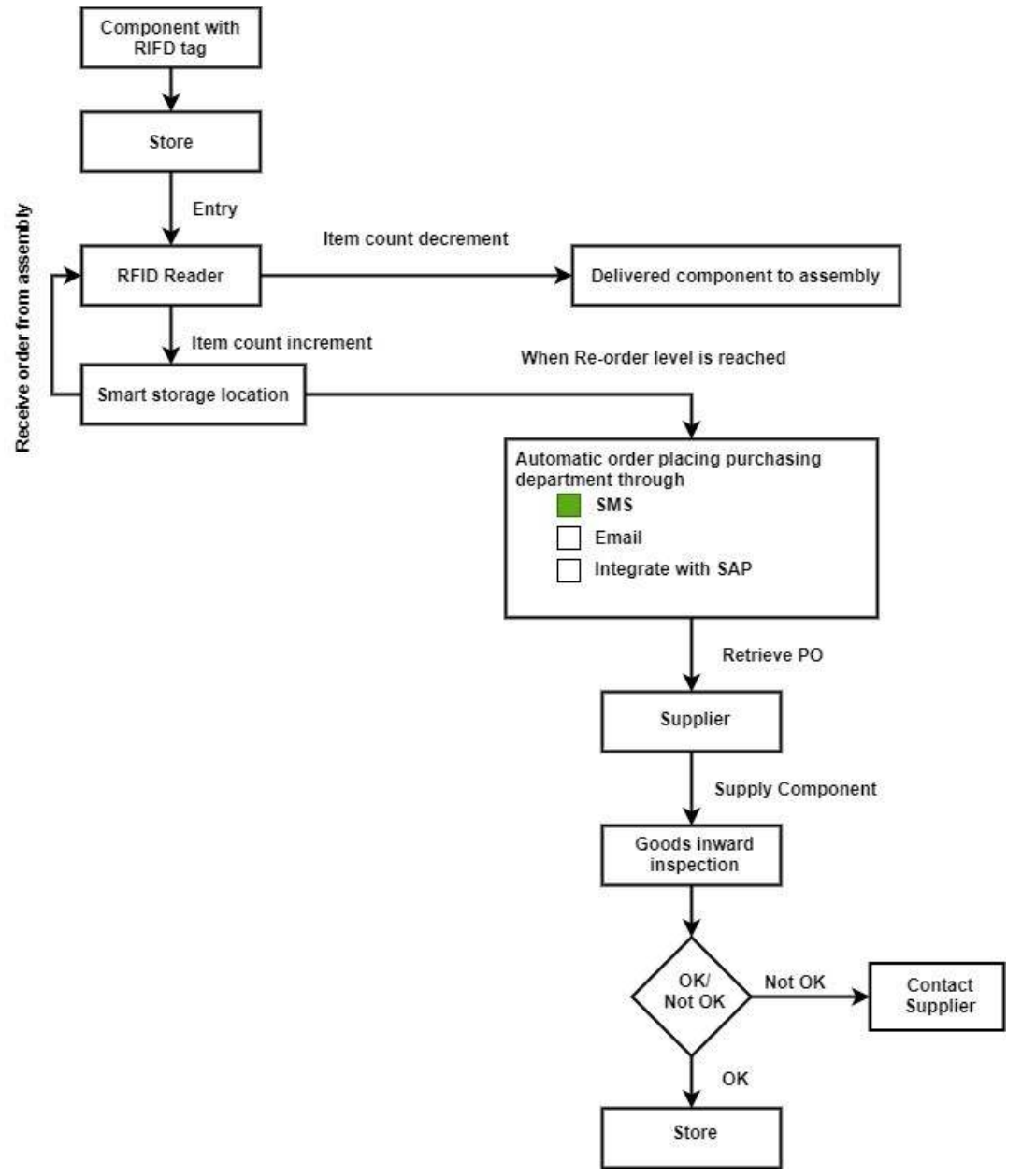

Fig. 3. Stock Model 


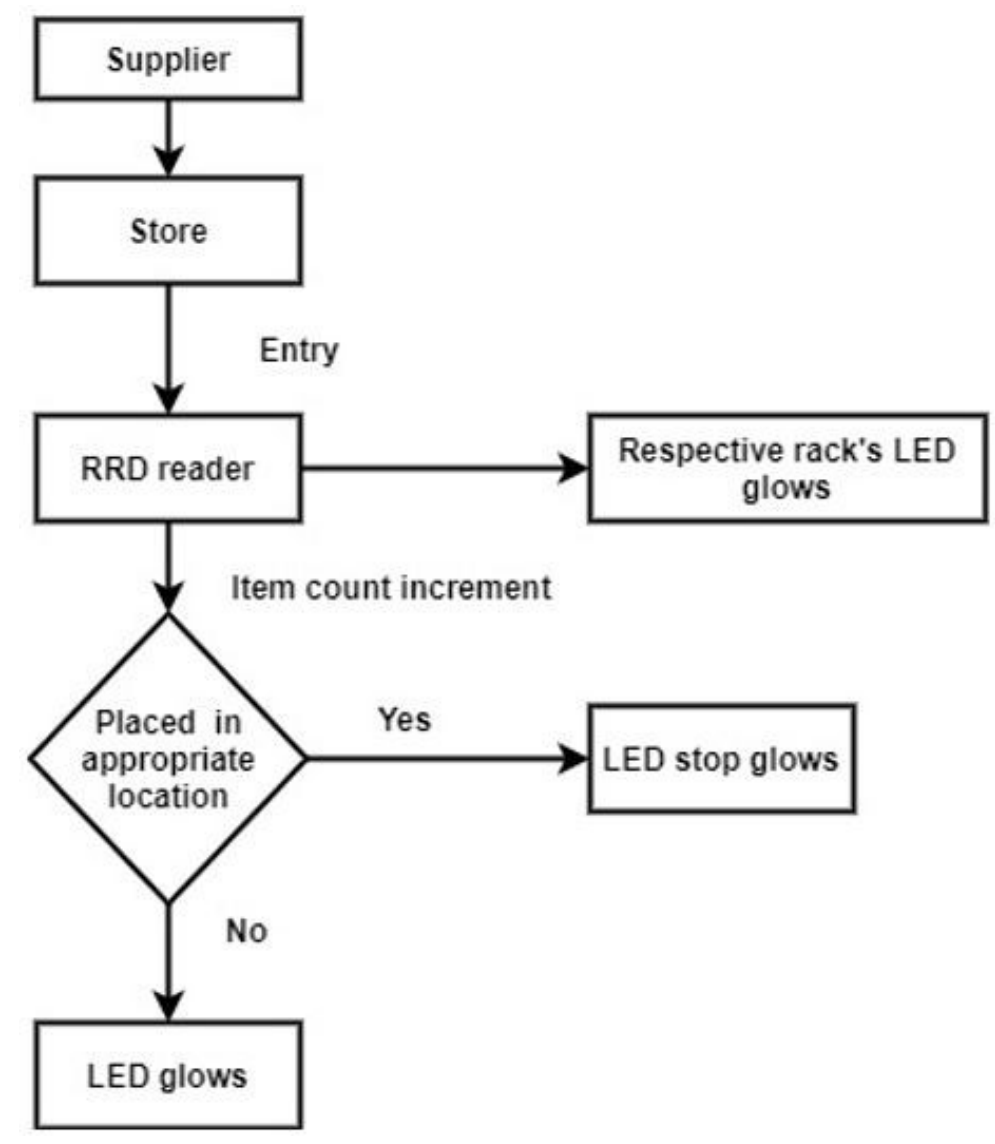

Fig. 4. Locate Model

RFID reader reads tag that is placed on medicine bins and then LED bulb in that respective cell will glow. 


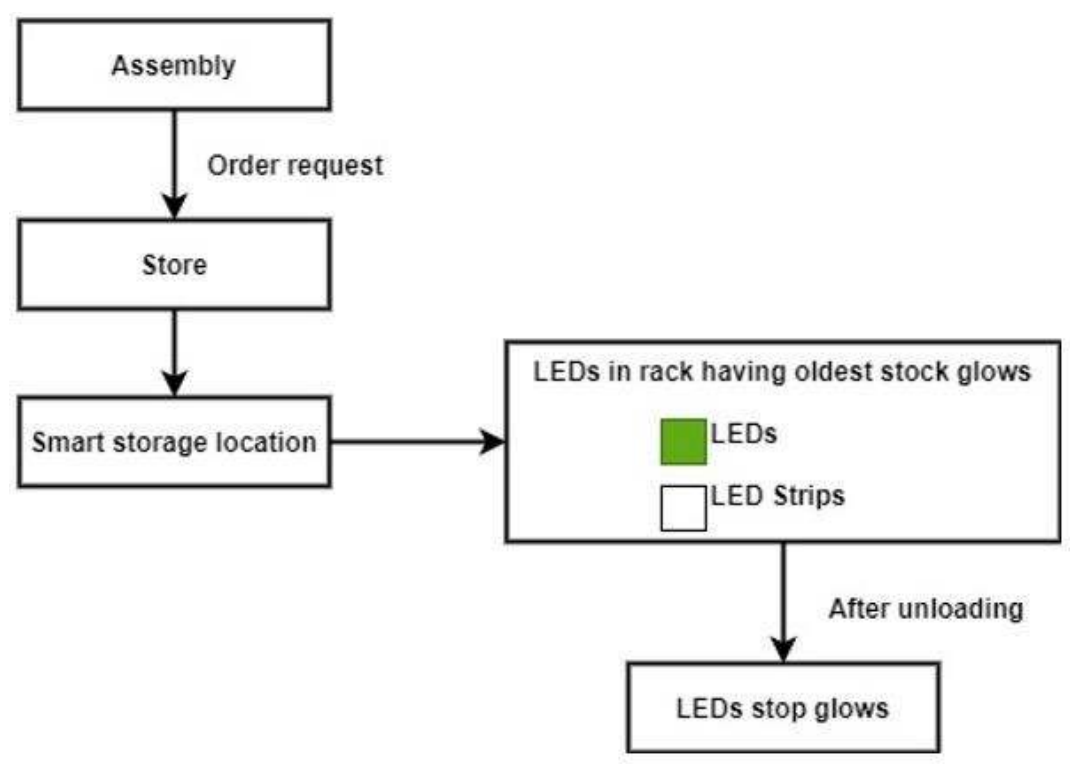

Fig. 5. FIFO Model

\subsection{FIFO Module}

The LED bulb will stop glows only when those respective medicines are placed in respective cells. Almost all the industries implement the FIFO (First-in and First-out) method because it saves money and time by calculating the exact cost of inventory sold, as the cost depends on most of the past cash flows from purchases that are used first. The FIFO module with ultrasonic sensors and LEDs is made use of for this purpose as given in figure 5.b Based on the literature collected in relevance to the proposed article, a PUGH Matrix was made to select the best out of the possible solution.

Matrix was used to select the possible technologies among the available for the smart kanban system. The datum is set to zero, and the technology that received score more than that of a datum, or that receives the highest score will be chosen to be used in the project.

The significance value was given based on the market research and expert advice through questionnaire. PUGH Matrix paves the way for effective and efficient decision making in selection of components. Based on the PUGH Matrix created it is safe to say that a RFID system will serve our purpose very well. 
Table 2. PUGH Matrix - comparison of proximity, laser and RFID

\begin{tabular}{lcccc}
\hline Criteria & Datum Proximity sensor(significance) & Laser (significance) & RFID (significance) \\
\hline Low cost & 0 & -1 & -2 & 0 \\
Detect item & 0 & 1 & -1 & 2 \\
Detect if it is the right one & 0 & -2 & 1 & 2 \\
Maintenance / parts cost & 0 & -1 & -2 & -1 \\
Simplicity & 0 & 0 & -1 & 0 \\
Training & 0 & -1 & -1 & -1 \\
Positives & 0 & 2 & 3 & 6 \\
Negatives & 0 & 6 & 9 & 4 \\
Total & 0 & -4 & -6 & 2 \\
\hline
\end{tabular}

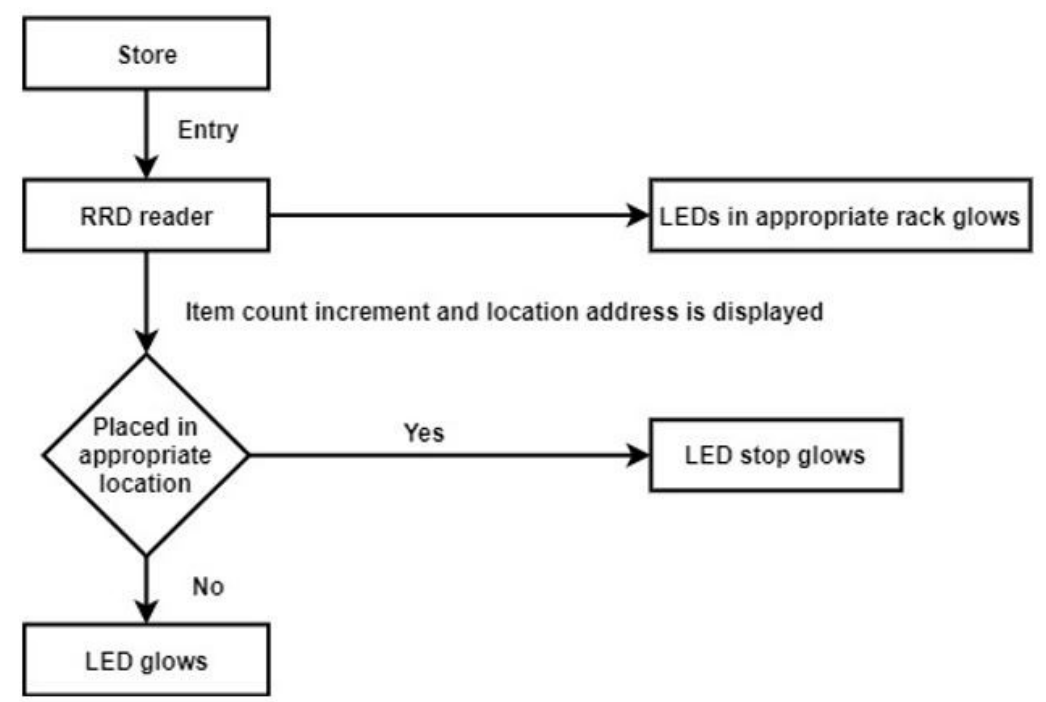

Fig. 6. Alert Model

Table 3. PUGH Matrix - comparison of PIC, Raspberry pi and Arduino

\begin{tabular}{lcccc}
\hline \multicolumn{1}{c}{ Criteria } & Datum PIC(significance) & Raspberry pi (significance) & Arduino (significance) \\
\hline Low cost & 0 & -18 & -2 & 1 \\
Libraries available & 0 & -1 & 1 & 2 \\
User friendly & 0 & -2 & 1 & 2 \\
Maintenance / parts cost & 0 & -2 & 1 & 1 \\
Simplicity & 0 & 0 & -1 & 0 \\
Program complexity & 0 & -1 & 0 & 1 \\
Positives & 0 & 0 & 3 & 4 \\
Negatives & 0 & 4 & 2 & 0 \\
Total & 0 & -4 & 0 & 7 \\
\hline
\end{tabular}


Based on the significance value and PUGH matrix, equipment used made and includes RFID reader-Low frequency (LF)RFID tag-passive, Arduino mega, GSM module - 900A and LCD. Initially the significance value is obtained from market research. Then the PUGH matrix is created which will help in the selection of equipment. After the selection of equipment, the prototype model is built and it comprises of Arduino Mega, LCD display, GSM module $100 \mathrm{~A}$, bread board, connecting wires, RFID RC-422 and RFID card tags. The prototype is tested successfully for all four modules and given in figure 7.

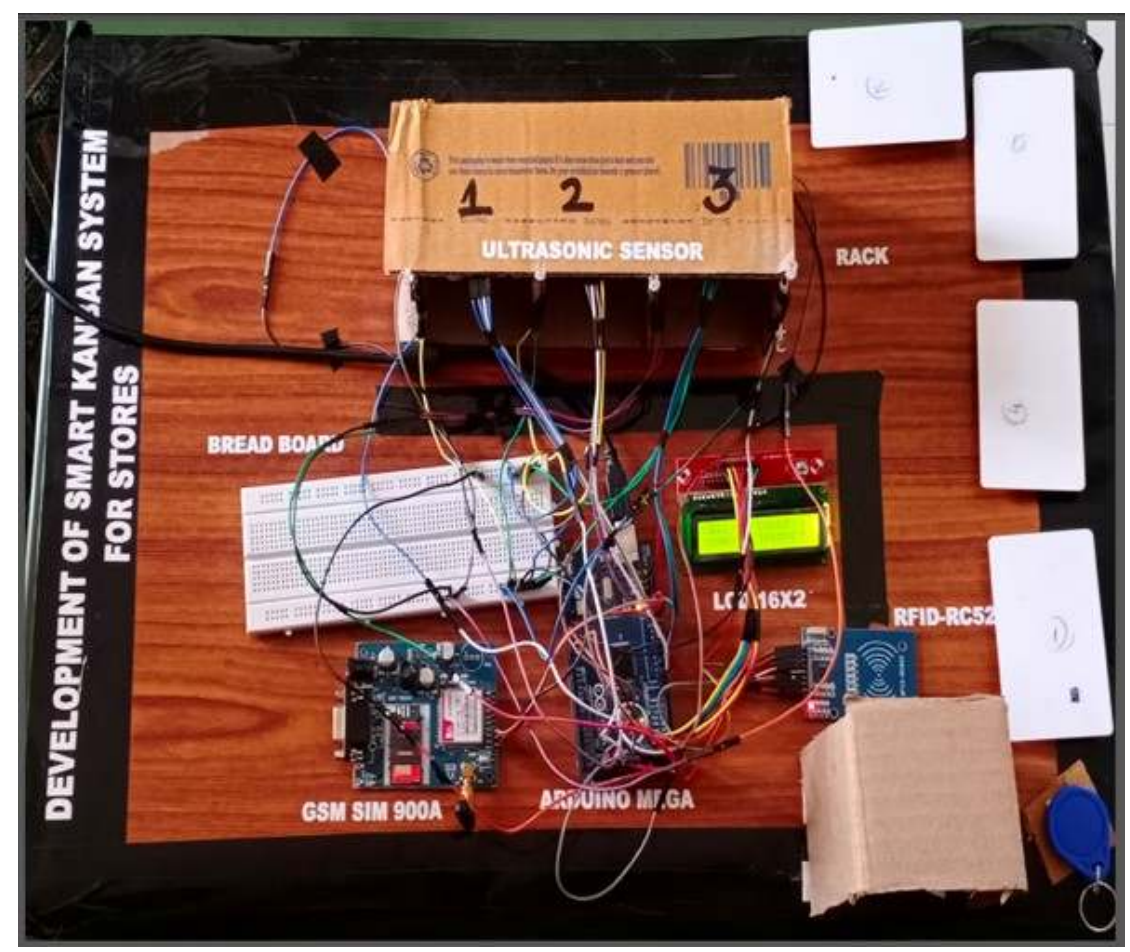

Fig. 7. Working Module of Smart Kanban based Pharmacy Stock Updation

The rack can be designed with mild steel along with Ultrasonic sensors located in the middle of the trays, which detect the medication based on distance and with the help of a scale and tape measure as given in figure 8 . 


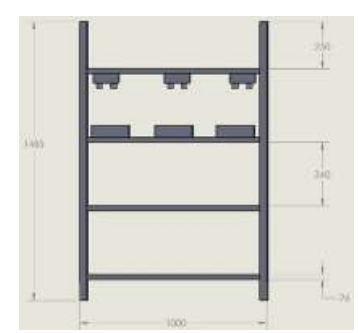

Fig. 8. Rack design

\section{Conclusion and Future Work}

A smart Kanban system that not only automatically places an order when the quantity reaches breakeven, but also maintains inventory data and eliminates manual inventory updates is developed. The developed system displays the right location for the medicine to be stored and also displays the path to reach that location and also helps the worker to find the first-in items during unloading the items for assembly. It can help the hospitals to store their oxygen cylinders and vaccines on a particular place. Based on the working of the prototype, future works are required on the system that would help industry for smooth supply chain flow. The future work can include a suitable cloud based smart kanban system with real time data could be created along with report generation and recommendations using Machine Learning techniques.

\section{References}

[1] N. Phumchusri and T. Panyavai, "Electronic kanban system for rubber seals production," Engineering Journal, vol. 19, no. 1, pp. 37-49, 2015.

[2] V. Ravichandran and G. K. N, "Implementation of kanban system for inventory tracking and establishing pull production," International journal of advances in production and mechanical engineering., vol. 1, pp. 31-37, 2015.

[3] K. Krishnaiyer, F. F. Chen, and H. Bouzary, "Cloud kanban framework for service operations management," Procedia Manufacturing,vol. 17, pp. 531-538, 2018.

[4] H. F. Abdulsada, "Design and implementation of smart attendance system based on raspberry pi," Journal of Babylon University/Engineering Sciences, vol. 25, no. 5, 2017.

[5] K. c. Kanvitha, Sirisha, "Timetable management system using raspberry pi and rfid," International Research Journal of Engineering and Technology (IRJET), vol. 9, no. 4, pp. 99$103,2017$.

[6] B. Mrugalska and M. K. Wyrwicka, "Towards lean production in industry 4.0," Procedia engineering, vol. 182, pp. 466-473, 2017.

[7] M. Tholkapiyan, A.Mohan, Vijayan.D.S , "A survey of recent studieson chlorophyll variation in Indian coastal waters", IOP Conf. Series: Materials Science and Engineering 993 (2020) 012041, doi:10.1088/1757-899X/993/1/012041.

[8] M. Lakshaga Jyothi et al., "Enabling intelligence through deep learning using IoT in a classroom environment based on a multimodal approach," Turkish Journal of Computer and Mathematics Education (TURCOMAT), vol. 12, no. 2, pp. 381-393, 2021.

[9] P. Lanza-Le'on, L. Sanchez-Ruiz, and D. Cantarero-Prieto, "Kanban system applications in healthcare services: A literature review," The International journal of health planning and management, 2021. 